Conclusion: IL-10 (-819 C/T, $-592 \mathrm{C} / \mathrm{A})$ and IFNY (+874 A/T) polymorphism might be related to RA in Japanese population. In addition, TGF $\beta_{1}$ (+869 A/T) polymorphism might be associated with the production of anti-CCP antibody. These results suggest that the analyzing cytokine gene polymorphisms may offer promise as useful factors in the choice of treatment for Japanese RA patients.

Table 1.

\begin{tabular}{|c|c|c|}
\hline $\begin{array}{l}\text { Cytokine } \\
G T^{*} \& \text { alleles }\end{array}$ & $\begin{array}{c}\text { RA } \\
(n=100)\end{array}$ & $\begin{array}{r}\text { Controls } \\
(n=50)\end{array}$ \\
\hline \multicolumn{3}{|l|}{ IFN $r+874$} \\
\hline $\mathrm{A} / \mathrm{T}$ & $21.0 \%(21 / 100) x^{2}=5.7736$ & $16.0 \%(8 / 50)$ \\
\hline $\mathrm{T} / \mathrm{T}$ & $0 \%(0 / 100))_{(p=0.0163)}$ & $6.0 \%(3 / 50)$ \\
\hline$A / A$ & $79.0 \%(79 / 100)$ & $78.0 \%(39 / 50)$ \\
\hline A allele & $89.5 \%(179 / 200)$ & $86.0 \%(86 / 100)$ \\
\hline T allele & $10.5 \%(21 / 200)$ & $14.0 \%(14 / 100)$ \\
\hline \multicolumn{3}{|l|}{$I L-10$} \\
\hline Genotypes & $x^{2}=78022$ & \\
\hline ACC/ACC (Lo) & $5.0 \%(5 / 100) \quad(p=0.0052)$ & $22.0 \%(11 / 50)$ \\
\hline ACC/ATA (Lo) & $43.0 \%(43 / 100)$ & $32.0 \%(16 / 50)$ \\
\hline ATA/ATA (L) & $46.0 \%(46 / 100)$ & $40.0 \%(20 / 50)$ \\
\hline GCC/ACC (Int) & $3.0 \%(3 / 100)$ & $1.0 \%(1 / 50)$ \\
\hline GCG/ATA (Int) & $2.0 \%(2 / 100)$ & $2.0 \%(2 / 50)$ \\
\hline GCC/GCC (H) & $0 \%(0 / 100)$ & O\% (0/50) \\
\hline$I L-10-819(\mathrm{C} / \mathrm{T})$ & $x^{2}=5.4162(0=0.0200)$ & \\
\hline $\mathrm{c} / \mathrm{C}$ & $8.9 \%(8 / 100)$ & $24.0 \%(12 / 50)$ \\
\hline $\mathrm{C} / \mathrm{T}$ & $45.6 \%(46 / 100)$ & $36.0 \%(18 / 50)$ \\
\hline $\mathrm{T} / \mathrm{T}$ & $45.6 \%(46 / 100)$ & $40.0 \%(20 / 50)$ \\
\hline C allele & $31.0 \%(62 / 200)$ & $42.0 \%(42 / 100)$ \\
\hline T allele & $69.0 . \%(138 / 200)$ & $58.0 \%(58 / 100)$ \\
\hline
\end{tabular}

References:

[1] Scott DL, Wolfe F, Huizinga TW. Rheumatoid arthritis. Lancet. 2010; 376: 1094-108.

[2] McInnes IB, Schett G. Cytokines in the pathogenesis of rheumatoid arthritis. Nat Rev Immunol. 2007 Jun;7(6):429-42.

Disclosure of Interests: None declared

Table 2.

\begin{tabular}{|c|c|c|c|c|}
\hline $\begin{array}{l}\text { Cytokine GT* } \\
\text { and alleles }\end{array}$ & $\begin{array}{c}\text { RA } \\
(n=100)\end{array}$ & $\begin{array}{c}\text { ACPA-positive } \\
(n=70)\end{array}$ & $\begin{array}{c}\text { ACPA-negative } \\
(n=30)\end{array}$ & $\begin{array}{r}\text { Controls } \\
(n=50)\end{array}$ \\
\hline TGF $\beta_{1}+869$ & & & ᄀ & \\
\hline $\mathrm{T} / \mathrm{C}$ & $52.0 \%(52 / 100)$ & $60.0 \%(42 / 70) x^{2}=$ & 8788 $33.3 \%(10 / 30)$ & $52.0 \%(26 / 50)$ \\
\hline $\mathrm{c} / \mathrm{C}$ & $28.0 \%(28 / 100)$ & $20.0 \%(14 / 70)$ (p $p=0$ & $4890) 46.7 \%(14 / 30)$ & $22.0 \%(11 / 50)$ \\
\hline$T / T$ & $20.0 \%(20 / 100)$ & $20.0 \%(14 / 70)$ & $20.0 \%(6 / 30)$ & $26.0 \%(13 / 50)$ \\
\hline $\mathrm{T}$ allele & $46.0 \%(92 / 200)$ & $50.0 \%(70 / 140)$ & $36.7 \times(22 / 60)$ & $52.0 \times(52 / 100)$ \\
\hline C allele & $54.0 \%(108 / 200)$ & $50.5 \%(70 / 140)$ & $63.3 \%(38 / 60)$ & $48.0 \%(48 / 100)$ \\
\hline
\end{tabular}

* : genotype

DOI: 10.1136/annrheumdis-2020-eular.2067

\section{FRI0551 PERFORMANCE OF THE 2019 AMERICAN COLLEGE OF RHEUMATOLOGY/EUROPEAN LEAGUE AGAINST RHEUMATISM SYSTEMIC LUPUS ERYTHEMATOSUS CLASSIFICATION CRITERIA}

C. Vrancianu ${ }^{1}$, I. Conea ${ }^{1}$, A. Boca', M. Bolboceanu ${ }^{1}$, C. Draganesscu', M. Sasu', C. Ciofu' ${ }^{1}$ L. Macovei ${ }^{1}$, M. Milicescu' ${ }^{1}$, M. Bojinca ${ }^{1}$, I. Ancuta', C. Mihai ${ }^{1}$, V. Stoica ${ }^{1}$, A. M. Gheorghiu ${ }^{1}{ }^{1}$ Cantacuzino Clinical Hospital, Carol Davila University of Medicine and Pharmacy, Internal Medicine and Rheumatology, Bucharest, Romania

Background: Systemic lupus erythematosus (SLE) is a heterogenous autoimmune disease, with increased morbidity and mortality, often diagnosed in advanced stages. The recently published 2019 American College Of Rheumatology/European League Against Rheumatism (ACR/EULAR) classification criteria for SLE are weighted, hierarchically clustered criteria developed to increase reliability and the identification of early SLE.

Objectives: To compare the sensitivity and specificity of the 2019 ACR/EULAR criteria with the 2012 SLICC criteria in a large single-centre cohort of patients with SLE, diagnosed according to expert oppinion.
Methods: Data of SLE patients evaluated in our centre between 1996-2019 have been retrospectively analyzed. The control cohort included patients with positive antinuclear antibodies of other ethiology than SLE, evaluated between 2001-2019. The sensitivity and specificity of the 2019 ACR/EULAR and 2012 SLICC criteria were tested using the McNemar test for correlated proportions.

Results: Four hundred and forty-six patients with SLE (413 women, mean \pm SD age $40.5 \pm 12.7$ years, disease duration $10.1 \pm 9.2$ years) and 67 controls (63 women, mean \pm SD age $50.4 \pm 12.6$ years, disease duration $7.6 \pm 6.9$ years; 29 systemic sclerosis (SSc), 18 mixed connective tissue dissease (MCTD), 15 undifferentiated CTD, 2 rheumatoid arthritis (RA), 2 SSc - RA overlaps and 1 dermatomyositis) were included. The sensitivity of the 2019 ACR/EULAR and 2012 SLICC criteria were similar $85.4 \%$ and $83.6 \%$, respectively $(p=0.3)$. The specificity of the 2019 ACR/EULAR and 2012 SLICC criteria were $70.2 \%$ and $86.6 \%$, respectively $(p=0.007)$. In the SLE group, patients missclassified according to the new 2019 ACR/EULAR criteria were 65, whereas according to the 2012 SLICC criteria were 73; of them, 44 patients did not fulfill any criteria. In the control group, patients misclassified had mainly MCTD (13/20 patients according to the new 2019 ACR/EULAR, and 8/9 according to the 2012 SLICC criteria).

Conclusion: In this real-life cohort, the 2019 ACR/EULAR criteria have a similar sensitivity and lower specificity than the 2012 SLICC criteria, misclassifying especially MCTD patients. These results might be due to the long disease duration in our cohort.

References:

[1] Aringer M, Costenbader K, Daikh D, et al. 2019 European League Against Rheumatism/American College of Rheumatology Classification Criteria for Systemic Lupus Erythematosus. Arthritis Rheumatol. 2019;71(9):1400-1412. doi:10.1002/art.40930

Disclosure of Interests: None declared

DOI: 10.1136/annrheumdis-2020-eular.3786

\section{FRI0552 GLOBAL, REGIONAL, AND NATIONAL BURDEN OF LOW BACK PAIN, 1990-2019: A SYSTEMATIC ANALYSIS FOR THE GLOBAL BURDEN OF DISEASE STUDY 2019}

D. $\mathrm{Wu}^{1}$, X. $\mathrm{Wu}^{1}$, J. Wu ${ }^{1}$, L. S. Tam ${ }^{2}$, J. Gu${ }^{1} .{ }^{1}$ Department of Rheumatology, The Third Affiliated Hospital of Sun Yat-Sen University, Guangzhou, China; ${ }^{2}$ Department of Medicine \& Therapeutics, The Prince of Wales Hospital, The Chinese University of Hong Kong, Hong Kong, Hong Kong (SAR)

Background: Low back pain (LBP) has become a major public health problem worldwide although the burden and underlying causes differ across locations and demographic groups.

Objectives: To report the distribution, trend and risk factor in the burden of LBP from the Global Burden of Disease Study 2019 (GBD 2019).

Methods: Based on GBD 2019, decomposition analyses were performed according to gender, age, geography and sociodemographic index (SDI). The number and age standardized rate of incidence, prevalence and disability adjusted life years (DALYs) with $95 \%$ uncertainty intervals (UI) were calculated.

Results: In 2019, female patients have a slightly higher number of prevalence $(17 \%)$, incidence $(15 \%)$ and DALYs $(16 \%)$ than male patients. Out of twenty 5-year age group, the number of incidences, prevalence, DALYs peak at 50-54 age group, while the rate of incidence, prevalence, DALYs peaked at 80-84 age group. From $5 \mathrm{SDI}$ regions, the highest number and age-standardized rate of incidence, prevalence, DALYs were observed in middle and high SDI region, respectively. Considering $21 \mathrm{GBD}$ regions, the highest number of incidence, prevalence, and DALYs were observed in East Asia, while the highest age standardized rate of incidence, prevalence and DALYs all found in Central Europe, High-income North America, High-income North America, respectively. In 204 countries and territories, the top 3 highest number of incidence, prevalence and DALYs were from China, India, United States of America. The top 3 highest age-standardized rate of prevalence, and DALYs were Georgia, United States of America, Denmark, while top 3 highest age-standardized rate of incidence were Poland, Vanuatu, Romania.

From 1990 to 2019, globally, the number of incidence, prevalence, DALYs increased by $50 \%, 47 \%, 47 \%$ to $223,738,363$ (95\%UI 197,935,799-253,300,243), $569,089,727 \quad(95 \%$ UI $505,632,980-641,256,710), \quad 63,533,528 \quad(95 \%$ UI $44,883,714-84,975,210)$, while age standardized rate of incidence, prevalence and DALYs decreased by $13 \%, 16 \%, 16 \%$ to 2,750 (95\%UI 2,427-3,108), 6,974 (95\%UI 6,192-7,862), 778 (95\%UI 548-1,043). In 5 SDI regions, low SDI region has the highest percentage increases in number of incidence, prevalence and DALYs, the highest percentage decrease in age standardized rate of incidence, prevalence and DALYs were observed in High-middle SDI. In 21 GBD regions, the highest percentage increase in number of incidence, prevalence and DALYs 